Monatsschrift f. Geburtshülfe u. Gynäkologie 1932;91:I-IV

\title{
Contents, Vol. 91, 1932
}

\section{Inhaltsverzeichnis.}

Seite

*Vorspruch [zur Festschrift fur Ludwig Seitz]. Von A.Martin-Berlin . 145

Originalarbeiten.

Albrecht, Hans, Operation und Kreislaufschädigung 56

*-Indikationsstellung zur digitalen Erweiterung des Muttermundes . 182 Bends, K., Über

Karzinom des Collum uteri nach vorausgegangener

Korpusamputation 79

Bucura, C, Die psychiatrische undneurologische Indikation zurSchwangerschaftsunterbrechung 22

Dawydow, G-. L., Über Gebärmutterausschabung zwecks Schwangerschaftsunterbrechung bei fehlender intrauteriner resp. nicht diagnostizierter extrauteriner Gravidität $\quad 447$

*Döderlein, A., 1st das Zurücklassen eines Fremdkörpers in der Bauchhöhle als fahrlässige Körperverletzung strafbar? Aus einem Obergutachten 147

*Dyroff, K., Der Mechanismus der Eiabnahme beim Menschen und seine Störungen 287

*Ehrhardt, K., Tierexperimentelle Untersuchungen über den Einfluß der

Milzexstirpation auf die Genitalfunktion und auf die Nachkommenschaft 323 *Eufinger, H., Das retikulo-endotheliale System in der Gestationsperiode

und im mensuellen Zyklus 312

*Frey, E., siehe Walthard, M.

Fried, Carl, Ungewöhnliche Reaktion bei Karzinombestrahlung ... 84

*Gänßbauer,. H., Siebenjälirige Erfahrungen über Eklampsieverhütung 268

G-ilmer, L·., Ûber k.ünstliche Scheidenbildung 48

*Gustafsson, L., Über die Abhängigkeit der klimakterischen Erscheinungen von der Art der Bestrahlung bei der B,öntgenbehandlung der Myome 238

*Guthmann, H., und May, W., Weitere Untersuchung zur Frage der intrauterinen.Nierenfunktion 306

G-ymnich, F., Über Leberverletzungen Neugeborener nach Spontangeburten 31

*Hilpert, F., Über Dickdarmstenose infolge entzündlicher Erkrankungen

der weibliehen Beckenorgane 279

Hochloff, A. W., Zur Histochemie der Whartonschen Sulze 381

Huhs, Enno, Die Intrakutanreaktion mit Ferrizyankali bei Icterus neonatorum $\quad 386$ 
*Jäger, Franz, Frühgeburt, Frühsterbliclikeit, Schwangerenfürsorge . 191 *K eh r e r , E., Über Akromegaloidismus und hypophysär-zerebrale Adipositas

bei einer Schwangeren $\quad 172$

Keii, Werner, siehe Legiehn, Herbert.

Kirsch, W., siehe Unterberger, F.

*Kolde, W., Über Spätrezidive bei Strahlenbehandlung des Gebärmutter-

krebses 214

Kovács, Franz, Beitrag zur Pathologie des Hirsutismus und Virilismus 65 Kückens, H., Beitrag

zur vergleichenden Morphologie an Plazenten von

verschiedenen Geburten bei derselben Frau 432

Landeker, Alfons, Frauenkunde und synoptische Diagnose in der

Gynäkologie 411

Legiehn, Herbert, Über die angebliche Potenzierung bei Kombination

von Wehenmitteln. Mit einem Nachtrag von Werner Keil, Königs-

berg i. Pr ... 436

Liebmann, Stephan, Über den Verlauf der mit Diabetes komplizierten

Schwangerschaften und Geburten 398

Die mit * bezeichneten Arbeiten sind Herrn Geheimrat Prof. Dr. L. Seitz in Frankfurt a. M. zum

60. Geburtstage gewidmet.

Liegner, B., Pyometra und Diabetes 91

IV

Inhaltsverzeichnis.

Seite *M ay, W., siehe Guthmann, H.

Mayer, A., Bemerkungen zur Frage der Thrombose und Embolie ... $39 *$ Mühlhausen, Lina,

Beitrag zur Frage der ovariellen Röntgen-Reiz-

bestrahlung 257

*Sarter, Ursula, Zur Frage des Einflusses der Bodenfeuchtigkeit auf

die Krebsentstehung 251

Schepetinsky, Anna, Krebsmetastase im Ovarium nach Magenresek-

tion wegen Karzinom 457

Seitz, Ludwig, Der Röntgenapparat neben dem Kreißsaal 453

de Snoo, K., Die Bedeutung der präventiven Geburtshilfe für Mutter

und Kind 1

*Spiegler, E., Lues und Schwangerschaft 340

Unterberger, F., und Kirsch, W., Bericht über Versuche zur Beein-

flussung des Geschlechtsverhältnisses bei Kaninchen nach Unterberger

(I. Mitteilung) 17

*Walthard, M. und Frey, E., Die neuzeitliche exspektative Geburts-

leitung und die Bedeutung der Wehenzählung als Grundlage für ihre

Begrenzung 157

*Wintz, H., Agomensin und Sistomensin 224

*Wolf-Jacob, Elsbeth, Rarzinombehandlung und praktischer Arzt . 460

Grenzgebîete für Geburtshilfe und Gynäkologie.

Physikalische Heilmethoden einschließlich der Hydrotherapie (II). Jahres-

bericht 1931. Von Oberarzt Hermann F r a n k e n-Freiburg i. Br. . 97 
Lebererkrankungen, uropoetisches System und Nierenerkrankungen in ihren Beziehungen zur Gynäkologie und Geburtshilfe. Von W. Bicken-

bach-Bonn 365

Zirkulationsapparat (II). Von Privatdozent Dr. A. Pfleiderer-Tübingen 464

Original-Sitzungsberichte aus geburtshilflich-gynäkologischen Gesellschaften.

Gynäkologische Gesellschaft zu Breslau. Sitzung vom 26. Januar 1932 . . 104 Gesellschaft für Geburtshilfe und Gynäkologie zu Berlin. Sitzung vom

27. November 1931 Ill

- $\quad$ Sitzungen vom 11. Dezember 1931, 22. Januar, 29. Januar, und 12. Februar 1932 507

Münchner Gynäkologische Gesellschaft. Sitzung vom 18. Juni 1931 . . 375

- $\quad$ Sitzung vom 25. Februar $1932 \quad 502$

Bayrische Gesellschaft für Geburtshilfe und Frauenheilkunde. Sitzung vom

15. Februar 1932

115

Niederrheinisch-Westfälische Gesellschaft für Gynäkologie und Geburts-

hilfe._96. Sitzung am 31. Oktober 1931 zu Düsseldorf

494

Geburtshilflich - gynäkologische Gesellschaft in Wien. Sitzung vom 9. Fe

bruar 1932 138

Sitzungen vom 8. März, 12. April und 10. Mai 1932

529

Streiflichter vom 56. Deutschen Chirurgenkongreß. Von Dr. G. H. Schnei der-Brandenburg a. H 534

Buchbesprechungen 140,377, 536

Perso $\pi$ alien $144,380,536$ 